\title{
INTERPRETACIÓN, TRADUCCIÓN Y COMUNICACIÓN, VARIEDADES DE LA INCONMENSURABILIDAD
}

\section{INTERPRETATION, TRANSLATION AND COMMUNICATION, VARIETIES OF INCOMMENSURABILITY}

\section{Pablo Melogno $a^{a^{*}}$}

Fechas de recepción y aceptación: 28 de septiembre de 2019 y 29 de abril de 2020

Resumen: En 1983 Thomas Kuhn introdujo la distinción entre interpretación y traducción, con el propósito de fortalecer la tesis de la inconmensurabilidad y responder a objeciones formuladas por Donald Davidson y Hilary Putnam. A partir de este contexto de debate, este trabajo revisa la distinción kuhniana con el objetivo de explorar las conexiones que pueden establecerse entre las teorías rivales y las comunidades científicas que las defienden. Para ello examino el papel de la traducción, la interpretación y la comunicación en el cambio teórico, con el propósito de ampliar la distinción de Kuhn y ahondar en algunas consecuencias no contempladas en su planteo original. Concluyo que la distinción entre estos planos lingüísticos resulta fundamental para comprender y evaluar el alcance de las divergencias suscitadas en casos de inconmensurabilidad.

\footnotetext{
a Departamento de Teoría, Facultad de Información y Comunicación, Universidad de la República.

* Correspondencia: Universidad de la República. Departamento de Teoría. Facultad de Información y Comunicación. San Salvador 1944, 11200 Montevideo. Uruguay.

E-mail: pablo.melogno@fic.edu.uy

El autor quisiera agradecer a Howard Sankey, Eric Oberheim, Leandro Giri y Hernán Miguel las discusiones e intercambios que impulsaron la redacción de este trabajo. También a los evaluadores anónimos de SCIO cuyos comentarios contribuyeron a mejorar sustantivamente la versión final, y a Leticia Camejo por la lectura detenida del texto.
} 
Palabras clave: Kuhn, inconmensurabilidad, interpretación, traducción, Putnam, Davidson.

Abstract: In 1983 Thomas Kuhn introduced the distinction between interpretation and translation, with the purpose of strengthening the incommensurability thesis and responding to objections made by Donald Davidson and Hilary Putnam. Departing from this debate, this paper examines the Kuhnian distinction, with the aim of exploring the connections which can be established between rival theories and the scientific communities which have adopted them. In order to do this, I will examine the role of translation, interpretation and communication in theoretical change, with the purpose of widening Kuhn's distinction and delving into some consequences not considered in his original stance. I will conclude by stating that the distinction between these linguistic domains is fundamental for understanding and evaluating the scope of the divergences generated in cases of incommensurability.

Keywords: Kuhn, incommensurability, interpretation, translation, Putnam, Davidson.

\section{$\S 1$ INTRODUCCIÓN}

Este trabajo se propone revisar algunas consecuencias de la tesis de la inconmensurabilidad de las teorías científicas introducidas por Thomas Kuhn (1962) en el contexto del debate suscitado entre Kuhn y algunos de sus críticos como Hilary Putnam, Donald Davidson, Mary Hesse y Harvey Siegel. Entiendo que el examen de este debate a la luz de la distinción kuhniana entre interpretación y traducción permite efectuar algunas distinciones relevantes sobre los conflictos semánticos vinculados a la tesis de la inconmensurabilidad. Espero mostrar que esto resulta de interés para abordar un grupo bastante heterogéneo de procesos lingüísticos que en el debate sobre la obra de Kuhn no pocas veces han sido tratados de forma indiscriminada.

El volumen de literatura secundaria sobre Kuhn generado en el último medio siglo es inmenso y se ha vuelto prácticamente inabordable. Con esta sobreabundancia de producción, no es tarea fácil justificar la pertinencia y novedad de ciertas discusiones, menos aún si se toma como punto de partida 
un debate desarrollado hace más de treinta años. Considero sin embargo que algunos de los malentendidos que trato de desanudar en este texto están aún presentes en las discusiones sobre el trabajo de Kuhn, y en la medida en que ejercen una influencia conceptualmente nociva su revisión resulta justificada.

La tesis de la inconmensurabilidad formulada por Kuhn (1962) ha sido uno de los tópicos más polémicos de la filosofía de la ciencia desde la segunda mitad del siglo xx hasta la actualidad. Una de sus principales dimensiones refiere al cambio lingüístico que se opera en las teorías que protagonizan una revolución científica. Durante una transición entre teorías inconmensurables -entiende Kuhn-, no solo se modifican las afirmaciones que se introducen sobre la naturaleza, sino que cambia también el significado de los términos empleados en dichas afirmaciones. De este modo, dos teorías inconmensurables no difieren solamente en cómo se comporta el mundo sino también en el significado de los términos que emplean. Hay términos que tienen significado en una teoría pero no en la teoría rival, y términos que son significativos en ambas teorías pero con significado diferente en cada una. En una nomenclatura que se ha vuelto estándar, estos fenómenos de variación de significado han sido agrupados por Sankey y Hoyningen-Huene (2001) bajo el rótulo de inconmensurabilidad semántica.

Esta perspectiva acarrea una larga lista de consecuencias que fue señalada por Kuhn. Primeramente, no es posible dirimir las diferencias semánticas entre teorías inconmensurables apelando a la observación, ya que los términos observacionales también están afectados por la variación de significado. Esto permite poner en cuestión la distinción misma entre términos teóricos y observacionales, y afirmar que no hay un lenguaje teóricamente neutro en el cual resolver las diferencias de significado. De aquí que no es posible formular en un único lenguaje las consecuencias contrastables de dos teorías rivales (Pérez Ransanz, 2012). Como han señalado Sankey (2000) y Rivadulla (2012), la inconmensurabilidad involucra una teoría contextual del significado, en la que todos los términos obtienen su significado de las teorías a las que pertenecen, por lo que, aunque el mismo término aparezca en dos teorías rivales, su significado no es el mismo en cada contexto teórico.

En segundo término, no es posible verter los términos de las teorías rivales en un tercer lenguaje que permita establecer correspondencias entre los 
términos que han variado de significado. Buena parte de las afirmaciones que se efectúan en una teoría dada no son formulables en una teoría rival, por lo que los lenguajes de dos teorías inconmensurables resultan intraducibles, al menos en los sectores en que hay variación de significado.

En tercer lugar, el enfrentamiento entre científicos que defienden teorías rivales carece de significados compartidos, lo que genera quiebres en la comunicación e imposibilita el desacuerdo racional. En estos términos, el debate entre defensores de teorías inconmensurables se torna un diálogo de sordos: "En el seno de los nuevos paradigmas, los viejos términos, conceptos y experimentos entran en nuevas relaciones mutuas. El resultado inevitable de ello es lo que podríamos llamar... un malentendido entre las dos escuelas rivales" (Kuhn, 1962: 309). La ausencia de acuerdo en el significado de los términos arrastra consigo divergencias sobre el dominio referencial, de modo que durante una revolución los científicos rivales no solo difieren sobre el significado de términos como masa o planeta, sino que tampoco concuerdan sobre la forma en que dichos términos fijan su referencia y se aplican en la naturaleza.

Por último, afirma Kuhn que el cambio en las estructuras conceptuales implica una reorganización en los procesos perceptivos. Dicho en otras palabras, poseer un nuevo vocabulario implica ver el mundo de un modo diferente, "los copernicanos que negaban al Sol el título tradicional de "planeta" no solo aprendían qué significa "planeta", o qué era el Sol. Sin duda estaban cambiando el significado de "planeta" a fin de que pudiera seguir estableciendo distinciones útiles en un mundo en que todos los cuerpos celestes, y no solo el Sol, se veían de modo distinto a como se habían visto antes..." (Kuhn, 1962: 281).

Al avanzar la década de los setenta, Kuhn $(1969,1970,1977)$ fue aumentando su interés por los problemas semánticos y lingüísticos de la inconmensurabilidad en desmedro de las cuestiones históricas y metodológicas. El resultado fue un énfasis en la inconmensurabilidad entendida como imposibilidad de traducción. Al mismo tiempo dedicó varios esfuerzos a moderar las consecuencias relativistas e irracionalistas atribuidas a su trabajo. Ello mediante la consideración de la inconmensurabilidad como un proceso localizado en ciertos sectores del cambio teórico, y la exploración de las posibilidades de comprensión mutua y desacuerdo racional entre teorías inconmensurables. 
En este contexto irrumpen las objeciones a la tesis de la inconmensurabilidad formuladas por Donald Davidson y Hilary Putnam, así como las correspondientes réplicas ofrecidas por Kuhn. En la sección 2 reviso estas objeciones, haciendo énfasis en los problemas vinculados a la traducción y al reconocimiento de la conducta lingüística tal como fueron planteados por Davidson-Putnam. En la sección 3 presento la respuesta de Kuhn y las razones que lo llevan a introducir la distinción entre traducir e interpretar. La sección 4 aborda algunas objeciones a Kuhn introducidas por Mary Hesse y Harvey Siegel. Introduce una distinción entre traducción, interpretación y comunicación, afirmando que esta distinción permite fortalecer la propuesta de Kuhn y que no está adecuadamente contemplada en las objeciones de sus críticos. La sección 5 está dedicada a explorar las relaciones entre estos tres conceptos, con el propósito de mostrar que refieren a procesos independientes con distinto nivel de incidencia en el cambio teórico. La conclusión defiende que varias de las dificultades que se han atribuido a la propuesta de Kuhn pueden despejarse bajo una adecuada distinción entre traducción, interpretación y comunicación.

\section{§2. Davidson, Putnam y los PROBlemas de LA TRAducción}

En De la idea misma de un esquema conceptual (1974), Davidson cuestiona algunas derivaciones de la tesis según la cual los esquemas conceptuales son las unidades básicas del conocimiento. Toma a los paradigmas kuhnianos como un caso de esquematismo e introduce algunas objeciones que atacan la tesis de la inconmensurabilidad. Para Davidson, la postulación de esquemas conceptuales quita inteligibilidad a nuestra explicación de las condiciones en que un sujeto comprende una lengua, en cuanto la traducibilidad es condición del reconocimiento de un lenguaje:

es dable decir que nada podría considerarse evidencia de que alguna forma de actividad no puede ser interpretada en nuestro lenguaje sin ser al mismo tiempo evidencia de que esa forma de actividad no puede ser una conducta de habla. Si esto fuera correcto, probablemente nos veríamos obligados a sostener que una forma de actividad que no puede interpretarse como lenguaje en nuestro lenguaje no es conducta de habla (1974: 191). 
El punto de Davidson es fuertemente intuitivo: supóngase que dos lenguajes naturales dados son intraducibles, para el caso, el hopi y el castellano. Si el lenguaje del hopi fuera totalmente intraducible al castellano, un hablante del castellano podría legítimamente dudar de que el hopi esté efectuando una conducta de habla cuando lo escucha emitir sonidos por su boca. Y si por el contrario, cuando escucha al hopi emitir sonidos por su boca los reconoce como un lenguaje, es que ya ha operado un nivel mínimo pero significativo de traducción.

Algún tiempo después Putnam introdujo una objeción similar: si aceptamos la inconmensurabilidad kuhniana, sería imposible interpretar cómo seres hablantes a aquellos que tienen teorías o visiones del mundo inconmensurables con las nuestras. Pero si por el contrario, consideramos los sonidos que emiten como un lenguaje dotado de sentido que puede ser descrito en nuestro lenguaje actual, entonces sus teorías no son inconmensurables con las nuestras: "Por muy diferentes que sean nuestras imágenes del conocimiento y nuestras concepciones de la racionalidad, compartimos un vasto fondo de suposiciones y creencias acerca de lo que es razonable incluso con la cultura más rara que consigamos interpretar" (1981: 124).

Davidson señaló también que, si los lenguajes de las teorías científicas del pasado son ininteligibles a ojos modernos, no se entiende cómo el trabajo del historiador de la ciencia nos ofrece una reconstrucción en nuestro lenguaje moderno de las teorías científicas del pasado. Si la inconmensurabilidad entraña algún tipo de dificultad profunda en la comprensión de lenguajes diferentes al nuestro, ¿cómo asegurar que el trabajo interpretativo del historiador con los textos científicos del pasado no presenta el mismo nivel del malentendido que separa a quienes poseen lenguajes inconmensurables? ${ }^{1}$

El alcance de estas críticas se comprende mejor teniendo en cuenta que para Putnam (1982) la racionalidad siempre se expresa en elementos históricos propios de un esquema conceptual pero no se agota en los elementos internos de cada esquema, ya que la definición de estos elementos presupone una idea de racionalidad. Esto es, si frente a una cultura diferente a la nuestra

\footnotetext{
${ }^{1}$ Esta orientación crítica fue retomada posteriormente por Philip Kitcher (1978) y Larry Laudan (1996).
} 
asumimos que no hay criterios comunes de racionalidad, lo que para nosotros cuenta como racional no podría aplicarse al comportamiento de los miembros de la otra cultura, por lo que su comportamiento no solo no nos resultará racional, sino que nos resultará directamente ininterpretable.

Supongamos que nos encontramos con un sujeto $S$ habitante de una cultura extraña, que comienza a emitir sonidos incomprensibles por su boca mientras hace algunos gestos. Si asumimos que nuestros criterios de racionalidad no valen fuera de nuestro esquema conceptual, no es lícito que introduzcamos frente a la conducta de $S$ elementos interpretativos tales como: a) los sonidos que está emitiendo $S$ pertenecen a su lenguaje; $b$ ) los está emitiendo con intenciones comunicativas; $c$ ) los gestos son un complemento de la emisión lingüística que también posee intención comunicativa, etc. Solo podemos asumir estos supuestos interpretativos en la comprensión de la conducta de $S$ si aceptamos el carácter trascendente -exterior a nuestro esquema conceptual-de algunos elementos imprescindibles para la interpretación.

Finalmente, la noción de esquema conceptual conduce para Davidson a un dualismo esquema-contenido, en el que aparece por un lado el lenguaje con sus categorías de organización de la experiencia, y por otro la experiencia misma que proporciona la materia prima a ser organizada. Este es el así llamado tercer dogma del empirismo, que a ojos de Davidson persiste en concepciones como las de Kuhn y Feyerabend. En estos términos, los fallos en la traducción indican cuándo estamos frente a esquemas diferentes, pero solo podemos afirmar que los elementos que están en juego cuando falla la traducción son esquemas dada su relación con un contenido empírico no organizado, "No podemos asignar un significado claro a la noción de organizar un único objeto, a menos que se entienda que el objeto contiene o consiste en otros objetos" (Davidson, 1974: 197).

La experiencia, los estímulos o lo que sea que es organizado por nuestros esquemas conceptuales, claramente no puede ser una unidad, sino que tiene que ser una pluralidad compuesta de partes a ser organizadas. El problema está en cómo identificamos dichas partes; si solo percibimos el mundo a través de nuestros esquemas conceptuales -y no hay experiencia posible fuera de cada esquema-, ¿qué es eso que percibimos solo a través de nuestros esquemas?, ¿qué es lo que nuestros esquemas organizan? Davidson muestra 
cómo la asunción de un mundo común tiene impacto en los problemas de la traducción. Si los estímulos $-\mathrm{u}$ otras entidades afines- son los mismos, y todos los lenguajes organizan la misma pluralidad de entidades, entonces no pueden ser tan diferentes entre sí como para generar fallos de traducción.

Davidson toma como principal blanco los fallos totales de traducción, es decir, los casos de pares de teorías rivales que no tienen términos con significado compartido. Para atacar los fallos parciales apela al principio de caridad, estableciendo que en el proceso de interpretar la conducta y las proferencias de un hablante debemos asumir como verdaderas la mayor parte de sus creencias, en cuanto el conocimiento de las creencias de los otros, así como el desacuerdo respecto de estas solo son posibles sobre una base de acuerdo común. Esto permite especificar los desacuerdos y las zonas de imposibilidad de la traducción.

Putnam (1981) avanzó en la misma dirección: de acuerdo al principio de caridad, la función de un esquema de traducción es interpretar las creencias de los demás, de modo que resulten razonables a la luz de lo que han pensado y experimentado. De acuerdo a esto, sin la apelación a la caridad no puede construirse ningún enunciado sobre la referencia de cualquier palabra proveniente de una época y un contexto conceptual diferente al nuestro. La palabra planta hace 200 años no incluía el concepto de fotosíntesis como sí lo incluye actualmente, del mismo modo que planeta hace 300 años no incluía a Urano y Neptuno, pero ello no impide que apelando a la caridad podamos dar cuenta de estos cambios en el marco de una traducción. En estos términos, el éxito interpretativo no requiere que las creencias que traducimos sean compatibles con las nuestras, sino solo que sean inteligibles.

Davidson razona que si las creencias de los demás no pueden ser masivamente erróneas, entonces el principio de caridad es condición para la interpretación ${ }^{2}$, mientras Putnam entiende que la caridad es condición para la interpretación si no queremos que las creencias de los demás resulten masiva-

2 "Dado que la caridad no es una opción, sino una condición para tener una teoría practicable, carece de sentido sugerir que en caso de respaldarla podríamos caer en un error generalizado" (Davidson, 1974: 202). 
mente ininteligibles ${ }^{3}$. La respuesta de Kuhn a estas objeciones no pasa-como frente a otros críticos- por moderar los desacuerdos o tratar de acercar las partes, sino por señalar que la argumentación de Davison-Putnam falla en cuanto confunde la interpretación con la traducción. Para Kuhn estos dos procesos son diferentes e irreductibles, y su especificación permite comprender mejor el tipo de ruptura lingüística que se produce entre dos teorías inconmensurables. En lo que sigue revisaremos la posición de Kuhn y algunas de sus consecuencias.

\section{§3. KUHN SOBRE TRADUCCIÓN Y VARIACIÓN DE SIGNIFICADO}

La respuesta de Kuhn a las objeciones de Davidson y Putnam es resultado de conjugar dos estrategias: la distinción entre interpretar y traducir un lenguaje y la restricción de la inconmensurabilidad a los fallos locales de traducción. Inicialmente Kuhn (1962) atribuyó al cambio conceptual un carácter abrupto y global, señalando que después de una revolución los científicos se enfrentan a un nuevo mundo. Sin embargo, su posterior consideración de la inconmensurabilidad en términos lingüísticos lo condujo a moderar algunas de sus tesis destacando el carácter local de los fallos de traducción ${ }^{4}$. En principio, la variación de significado y la relación de intraducibilidad se dan en sectores acotados del enfrentamiento entre teorías inconmensurables, que mantienen un amplio rango de conceptos compartidos:

La mayoría de los términos comunes a las dos teorías funcionan de la misma forma en ambas; sus significados, cualesquiera que puedan ser, se preservan; su traducción es simplemente homófona. Surgen problemas de traducción únicamente con un pequeño subgrupo de términos (que usualmente

\footnotetext{
3 “el éxito interpretativo no requiere que las creencias de las personas a las que se está traduciendo resulten ser nuestras mismas creencias, sino que nos resulten inteligibles. Esta es la base de las diversas máximas de caridad interpretativa o del «beneficio de la duda »"(Putnam, 1981: 195).

${ }^{4} \mathrm{Si}$ bien ya en el Epílogo a La Estructura Kuhn menciona que las teorías inconmensurables mantienen conceptos y criterios metodológicos comunes, es a lo largo de la década de los setenta (Kuhn, 1974, 1977) cuando ofrece una elaboración más desarrollada de la inconmensurabilidad local. Para la evolución posterior del relativismo lingüístico en Kuhn, es de relieve el trabajo de Mayoral (2017).
} 
se interdefinen) y con los enunciados que los contienen. La afirmación de que dos teorías son inconmensurables es más modesta de lo que la mayor parte de sus críticos ha supuesto (Kuhn, 1983: 99).

Una vez que la inconmensurabilidad es un fenómeno local la base de significados compartidos permite la comprensión mutua, por lo que tanto los riesgos de ininteligibilidad de la conducta no afectan esta formulación restringida: "Los términos que preservan sus significados a través de un cambio de teoría proporcionan una base suficiente para la discusión de las diferencias, y para las comparaciones que son relevantes en la elección de teorías" (1983: 100).

Frente a esto, aún puede insistirse en cómo es posible que los historiadores entiendan las teorías científicas del pasado o que dos científicos rivales logren comprenderse. Puede aceptarse a Kuhn que en ambos casos hay conceptos compartidos que permiten la comunicación y la comprensión. Pero al mismo tiempo puede afirmarse - contra Kuhn- que la comprensión se agota donde terminan los conceptos en común y que la comunicación no es posible frente a los fallos de traducción. ¿En qué medida los conceptos compartidos permiten lograr entendimiento en los sectores donde la traducción fracasa? ${ }^{5}$

La respuesta de Kuhn se basa en la distinción entre interpretación y traducción, conceptos que aluden a dos procesos diferentes que a su entender aparecen erróneamente fundidos en sus críticos. Una traducción supone un sujeto que ya conoce dos lenguajes y que trata de establecer equivalencias más o menos aproximadas entre los términos de cada uno. En el proceso no cambian los significados de las palabras en juego, ya que están dados previo a la traducción. La interpretación, por el contrario, supone un sujeto que domina una sola lengua y se enfrenta a otra que es desconocida:

A diferencia de la persona que traduce, puede que la que efectúa la interpretación domine inicialmente solo una lengua. Al principio, el texto sobre el que trabaja consiste total o parcialmente en ruidos o inscripciones ininteligibles. El "traductor radical" de Quine es, de hecho, alguien que efectúa una interpretación, y "gavagai" ejemplifica el material ininteligible con el que comienza (Kuhn, 1983: 104).

${ }^{5}$ Una interesante tentativa de responder a esta pregunta se encuentra en Falguera (2012: 148 y ss.). 
Cuando la interpretación tiene éxito, quien la lleva a cabo termina convirtiéndose en hablante del lenguaje interpretado, lo que convierte al bilingüismo en una estrategia eficaz que los científicos pueden activar frente a las divergencias semánticas que los separan. Si las teorías que experimentan variaciones de significado no están condenadas a situaciones de malentendido irreversible, es gracias a que algunos científicos desarrollan con éxito prácticas basadas en el aprendizaje de la teoría rival, "La imposibilidad de traducción no impide, desde luego, que los usuarios de un léxico aprendan el otro. Y habiéndolo hecho, pueden unir los dos a la vez, enriqueciendo su léxico inicial añadiéndole conjuntos de términos del otro que acaban de adquirir"' (Kuhn, 1989: 95).

Ahora bien -avanza Kuhn-, el éxito del intérprete en aprender una nueva lengua es independiente del grado en que el vocabulario de la lengua aprendida sea traducible a la lengua original del intérprete. El sujeto encontrará que algunos de los términos que ha aprendido tienen fácilmente un correlato en su lengua nativa, mientras que otros resultan intraducibles a esta. Pero si el sujeto ha aprendido con éxito los términos intraducibles, entonces puede utilizarlos de forma adecuada y comprender las aplicaciones de su uso, incluso aunque no pueda traducirlos a su lengua nativa: "Aprender una lengua no es lo mismo que traducir de ella a la propia. Tener éxito en lo primero no implica tener éxito en lo segundo" (Kuhn, 1983: 103).

La inconmensurabilidad entendida como intraducibilidad ocurre cuando un hablante sabe utilizar un término, pero para utilizarlo correctamente debe hablar el lenguaje que ha aprendido, ya que su lengua materna no ofrece un término equivalente. Esto implica que la interpretación tiene éxito donde la traducción fracasa ${ }^{6}$. La propuesta de Kuhn apunta a mostrar que buena parte de las objeciones introducidas por sus críticos responden a la indistinción entre la traducción entre dos lenguajes y la interpretación de un lenguaje por un sujeto. Entiendo que esta distinción permite clarificar de qué tipo de procesos lingüísticos puede predicarse la inconmensurabilidad y qué tipo de propiedades

\footnotetext{
${ }^{6}$ En palabras de Marcum, "el término no puede ser traducido, i. e. que no hay un equivalente del término en el lenguaje teórico competidor. En otras palabras, para que el término teórico tenga significado el científico debe volverse "nativo" en su uso" (2005: 123).
} 
son atribuibles a teorías inconmensurables. En la siguiente sección abordaré ambos problemas tomando como punto de partida la réplica de Mary Hesse a la distinción acuñada por Kuhn.

\section{§4. TRADUCCIÓN, INTERPRETACIÓN Y COMUNICACIÓN}

Mary Hesse introdujo rápidamente una contrarréplica a Kuhn, alegando que toda traducción supone la puesta en juego de principios interpretativos, "La traducción extensional es un ideal raramente realizado en la traducción real que generalmente requiere ser suplementada por principios hermenéuticos adicionales" (1983: 708). El punto de Hesse es que la interpretación y la traducción mantienen determinadas relaciones que impiden tomarlos como independientes. Pero incluso si se acepta que en todo proceso de traducción intervienen algunos principios interpretativos, esto no afecta a la idea de que interpretar y traducir son dos procesos diferenciados. Si como consecuencia de sus rasgos semánticos dos lenguajes resultan intraducibles, ningún principio interpretativo podrá tornarlos traducibles, ya que la relación de traducibilidad/intraducibilidad depende de las características semánticas de los lenguajes y no de las operaciones interpretativas de los sujetos. Del mismo modo, dados dos lenguajes que por sus características semánticas son traducibles, nuestra traducción puede fallar a causa de nuestra ignorancia o de un mal uso de ciertos principios interpretativos, pero ello no afecta el hecho de que nos hallamos frente a dos lenguajes traducibles.

La traducción supone una relación semántica entre dos lenguajes, en tanto requiere formular el contenido de un lenguaje con los conceptos de otro. Que esto pueda o no hacerse es resultado de las capacidades expresivas de cada lenguaje, más que de las estrategias interpretativas introducidas por los sujetos $^{7}$. Por otro lado, la interpretación -e incluso la introducción de los prin-

\footnotetext{
${ }^{7}$ Como ha señalado Falguera, la inconmensurabilidad supone una noción de traducción especialmente restrictiva: "La traducción en el sentido cotidiano es tentativa y bajo compromisos. La traducción estricta entre dos lenguas (o lenguajes de dos teorías) supone la sustitución sistemática de palabras de una lengua por otras de otra lengua preservando la equivalencia de contenido para los diferentes contextos de uso" (2012: 123).
} 
cipios interpretativos que permiten la traducción- es un proceso epistémico dado entre un sujeto y un lenguaje, por lo que se encuentra en un plano diferente a la traducción. Del fracaso o éxito en el plano semántico no puede inferirse el fracaso o éxito en el plano epistémico, ni viceversa. Por esta razón, el éxito de cada uno de estos procesos es independiente del éxito del otro.

Esta distinción es curiosamente afín a Michael Devitt, quien señaló tempranamente que en los debates sobre la inconmensurabilidad aparecían confundidos los problemas semánticos de las relaciones entre teorías y los problemas epistémicos de la justificación.

Considérese, por ejemplo, la afirmación de que los enunciados de observación están teóricamente determinados. Esto puede significar la tesis semántica (falsa a mi ver) de que el significado de un enunciado de observación está determinado por la teoría de la que se deriva. También puede significar la tesis epistémica (verdadera, a mi ver), de que el juicio sobre el valor de verdad de un enunciado de observación es parcialmente dependiente de las creencias de uno en varias teorías, que podrían ser falsas (Devitt, 1979: 32).

Si bien Devitt realizó esta formulación en un contexto de crítica a la tesis de la inconmensurabilidad, los problemas que señala afectan tanto las posiciones de Kuhn como las de algunos críticos. Entre varios ejemplos cabe tomar a Harvey Siegel, para quien las divergencias entre teorías inconmensurables impiden la comunicación significativa entre los científicos que las adoptan. A la inversa, si hay comunicación significativa, entonces no hay divergencias de fondo y por tanto no hay inconmensurabilidad:

Kuhn quiere sugerir que los dos científicos pueden deliberar significativamente sobre la fertilidad de sus respectivas teorías. Si Kuhn está dispuesto a permitir una deliberación tal... sus comentarios sobre los valores son claramente inconsistentes con mantener la inconmensurabilidad, ya que la inconmensurabilidad garantiza la imposibilidad de una deliberación semejante (Siegel, 1980: 365). 
La inconmensurabilidad es una tesis acerca de la relación entre dos estructuras teóricas, $v g r$. paradigmas, teorías o léxicos ${ }^{8}$. A partir de aquí es posible establecer conexiones entre la inconmensurabilidad dada entre las estructuras teóricas y lo que sucede en las prácticas comunicacionales de los científicos. Lo que no parece tan sencillo es, como pretende Siegel, inferir que la relación de inconmensurabilidad entre dos estructuras teóricas cancela determinadas prácticas comunicacionales. Los científicos que adoptan teorías inconmensurables pueden comunicarse mejor o peor según el caso, y pueden deliberar en mayor o menor medida dependiendo de las estrategias de comunicación y los problemas que estén en juego en cada debate. Pero la posibilidad (o imposibilidad) de interacción comunicativa no puede inferirse de la sola relación de inconmensurabilidad entre las teorías en juego.

En las objeciones de Putnam y Davidson la traducción aparece ligada a la posibilidad de reconocer una conducta como lingüística y de asignar significado a las proferencias de los hablantes de una lengua. En esa medida Kuhn estaba justificado en protestar que sus críticos no distinguían los problemas de la traducción y los de la interpretación. Una vez que consideramos la interpretación y la traducción como dos procesos categorialmente diferentes, los resultados obtenidos en uno no quedan ligados al otro. De este modo, la interpretación puede tener éxito donde la traducción fracasa. La objeción de Siegel por su parte no distingue entre las dificultades para traducir dos teorías y los problemas de comunicación que pueden tener los científicos que las defienden. Al igual que en la discusión Davidson-Putnam, esto supone que los resultados de los procesos de traducción determinan las posibilidades de comunicación. Esta idea se sostiene en la consideración de los procesos de comunicación como directamente dependientes de los rasgos semánticos de las teorías en juego. Esto es, si dos científicos adoptan teorías intraducibles entre sí, entonces no

${ }^{8}$ Kuhn (1981) introdujo el concepto de inconmensurabilidad taxonómica, considerando a las taxonomías como redes de términos interdefinidos que forman un léxico. El léxico define una serie de relaciones jerárquicas entre clases más un conjunto de criterios para incluir objetos dentro de las clases que cada término designa. La estructura de una taxonomía exige que no haya superposición entre dos clases, excepto que mantengan una relación de género y especie. Si bien no me detendré en la noción kuhniana de taxonomía, considero que las posiciones principales que aquí defiendo se aplican tanto a las taxonomías como a otras unidades de análisis (léxicos, esquemas conceptuales, lenguajes teóricos, paradigmas) que Kuhn utilizó a lo largo de su obra. 
podrán comunicarse. Pero si se considera a la comunicación como un proceso independiente de la traducción pueden obtenerse resultados diferentes.

Tanto Davidson como Putnam y Siegel buscan al mismo tiempo cuestionar el planteo de Kuhn y echar algo de luz sobre las diferencias lingüísticas que separan a los científicos que defienden teorías rivales. Pero un obstáculo para llevar adelante ambos objetivos es la presunción de que estas diferencias lingüísticas pueden ser reducidas a un mismo plano o agrupadas bajo una misma categoría semántica.

Una buena estrategia para desanudar estos problemas consiste en partir de que los científicos que defienden teorías rivales mantienen desacuerdos que se manifiestan en distintos niveles y que remiten a procesos lingüísticos de naturaleza muy diferente. Esto puede verse mejor si se adscriben los problemas de la traducción a las relaciones entre lenguajes teóricos, los problemas de la interpretación a las relaciones entre sujetos y lenguajes y los problemas de comunicación a las relaciones entre sujetos.

La traducción es un proceso que requiere de dos lenguajes, y que puede resultar fallido o exitoso en función de las características de dichos lenguajes. Un lenguaje solo puede ser considerado traducible $-\mathrm{o}$ intraducible- en relación con otro lenguaje. La traducción es exitosa cuando ambos lenguajes poseen rasgos semánticos tales que permiten producir oraciones con significado equivalente en uno y otro, y fracasa cuando la equivalencia no es posible. En estos términos, la traducción puede considerarse como una relación semántica entre dos lenguajes. Así las cosas, la traducción no requiere necesariamente un sujeto epistémico que medie en el proceso, una computadora adecuadamente programada podría indicarnos si un término de un lenguaje resulta o no traducible a otro.

La interpretación por su parte requiere de al menos un sujeto y un lenguaje, y puede resultar fallida o exitosa en función de las estrategias interpretativas desplegadas en cada caso por los sujetos, ya que no en función de las características del lenguaje interpretado. Trivialmente, la traducción es una relación simétrica-si un término es traducible del lenguaje $A$ al $B$, entonces es traducible de $B$ a $A$-, y la interpretación es una relación asimétrica, solo los sujetos interpretan lenguajes y no viceversa. En estos términos, la interpretación es exitosa cuando un sujeto aprende a utilizar correctamente los términos del 
lenguaje objeto de la interpretación, de forma que se termina convirtiendo en un hablante competente del dicho lenguaje.

Un lenguaje teórico es interpretable para un sujeto, no para otro lenguaje, por el solo hecho de que un lenguaje no es capaz de realizar interpretación alguna. De aquí que la afirmación de que dos lenguajes son intraducibles no puede equivaler a que son ininterpretables. La imagen putnamiana del reconocimiento de un lenguaje requiere un sujeto que interpreta y un lenguaje objeto de la interpretación, por lo que se trata de un proceso ajeno a la traducción de un lenguaje en otro, en cuanto la interpretación es una relación epistémica entre un sujeto y un lenguaje. Del mismo modo que un lenguaje es traducible o intraducible respecto de otro lenguaje, un lenguaje es interpretable o no interpretable para un sujeto.

En tercer término, pueden distinguirse los procesos de comunicación, vinculados por Siegel a los malentendidos entre científicos que defienden teorías rivales. Al contrario de la traducción -que requiere dos lenguajes-y la interpretación -que requiere un lenguaje y un sujeto-, la comunicación requiere necesariamente dos sujetos. Todo lo que es comunicable lo es de un sujeto a otro, por lo que la comunicación requiere un sujeto que tiene ciertas conductas de interacción comunicacional con otros sujetos. En estos términos la comunicación puede ser considerada como una relación que involucra la conducta lingüística de al menos dos hablantes.

Si esto es así, las fallas en la comunicación se sitúan en el nivel de las prácticas comunicacionales, y no aluden a las relaciones semánticas entre los lenguajes ni a las relaciones epistémicas entre el sujeto y el lenguaje. Dicho de otro modo, el que dos lenguajes sean intraducibles es independiente de que sus hablantes tengan o no problemas de comunicación, y a su vez dos hablantes de lenguajes traducibles pueden tener problemas de comunicación. Ello explica por qué dos lenguajes intraducibles pueden dar lugar a interacciones lingüísticas en que los hablantes fallan al comunicarse, junto a otras en las que logran hacerlo con éxito.

Las fallas en la comunicación frecuentemente asociadas a la inconmensurabilidad no se sitúan en el nivel semántico de las relaciones entre lenguajes ni en el nivel epistémico de las relaciones entre sujetos y lenguajes, sino en el nivel de las prácticas lingüísticas de los sujetos. En estas condiciones, una 
consecuencia de la confusión señalada por Devitt consiste en detectar que a nivel de las conductas lingüísticas los científicos tienen problemas de comunicación y atribuir la causa a las características semánticas de las teorías en juego. O viceversa, afirmar que si dos teorías son intraducibles sus respectivos defensores tendrán problemas de comunicación ${ }^{9}$.

Hasta aquí he defendido una distinción categorial entre traducción, interpretación y comunicación, con el propósito de mostrar que son procesos diferenciados, predicables de unidades diferentes y no reductibles entre sí. Ahora bien, esto no inhibe que puedan establecerse relaciones y condiciones de interacción entre estos tres planos. En lo que sigue trataré en explorar algunas de ellas.

\section{§5. LOS LÍMITES DE LA TRADUCIBILIDAD}

La clarificación de niveles lingüísticos que he ofrecido permite fortalecer la idea kuhniana de la inconmensurabilidad como relación de intraducibilidad entre teorías rivales, en la medida en que delimita con más claridad cuál es el dominio de los procesos de traducción, y a distinguir los problemas de la traducción de otros procesos dados en niveles lingüísticos diferentes. El problema inicial no afecta solo a los críticos sino al propio Kuhn, ya que por momentos Kuhn parece pensar - al igual que Siegel- que los fallos en la comunicación son una consecuencia inevitable de la inconmensurabilidad: "que quienes sostienen puntos de vista inconmensurables sean considerados como miembros de diferentes comunidades lingüísticas, y que sus problemas de

\footnotetext{
${ }^{9}$ Podría cuestionarse si la incorporación de este triple nivel de análisis no deflaciona el propio concepto de inconmensurabilidad, en cuanto que una vez identificadas las situaciones de intraducibilidad o los fallos en la comunicación no resultaría superfluo hablar de lenguajes inconmensurables. Nuestra argumentación supone una lectura deflacionista de la inconmensurabilidad en cuanto se enmarca en la versión local de la inconmensurabilidad acuñada por Kuhn (1983), en la que los fenómenos de intraducibilidad y variación de significado se localizan en ciertos sectores de las teorías en disputa. Sin embargo, la distinción no anula el concepto de inconmensurabilidad, en cuanto si dos teorías son inconmensurables -como consecuencia de sus divergencias semánticas- lo seguirán siendo con independencia de las estrategias interpretativas que desarrollen los sujetos o de las conductas lingüísticas que medien en la comunicación.
}

SCIO. Revista de Filosofia, n. ${ }^{\circ}$ 18, Julio de 2020, 87-111, ISSN: 1887-9853 
comunicación sean analizados como problemas de traducción” (Kuhn, 1970: 270).

No es mi propósito discutir las implicaciones de este tipo de pasajes en la inconmensurabilidad tal como Kuhn la defendía en la década de los sesenta. Entiendo que la versión local de la inconmensurabilidad ligada a los fallos de traducción que Kuhn ofrece en 1983 resiste de mejor manera la indistinción de niveles que su propio trabajo mostraba en la época de La Estructura. Esto en cuanto la inconmensurabilidad local implica que los fallos de traducción y las variaciones semánticas se localizan en ciertos sectores del conflicto teórico, lo que permite que dos teorías inconmensurables compartan ambas porciones de vocabulario y significados comunes. Esto impacta asimismo en los problemas de la interpretación y la comunicación, ya que los problemas de estos dos niveles también se desarrollan sobre esta misma base de contenido conceptual común a las teorías rivales. Una vez clarificado el dominio de los procesos de traducción, es posible explorar de mejor manera las relaciones entre dichos procesos y otros niveles lingüísticos.

Como han señalado Favretti, Sandri y Scazzieri (1999), la comunicación puede tornarse más dificultosa en ausencia de estándares lingüísticos compartidos, y las posibilidades de superar las dificultades dependerán de las estrategias que desarrollen los hablantes en cuestión. Cada lenguaje teórico fija coordenadas de comunicación entre sus hablantes, de modo que la comunicación plausiblemente será más fluida entre los científicos que hablan el mismo lenguaje que entre aquellos que hablan lenguajes diferentes.

Pero aún en este último caso, no es de recibo asumir las fallas en la comunicación como una consecuencia de las divergencias semánticas. Michael Friedman ha señalado que en épocas de ciencia normal la racionalidad comunicativa de la ciencia queda asegurada por la presencia de un paradigma que proporciona principios comunes de racionalidad. Pero durante una revolución científica estos principios se rompen, lo que genera rupturas en la racionalidad comunicativa: "los paradigmas sucesivos en una revolución científica son fundamentalmente no intertraducibles, y por tanto no comparten lo que sea necesario para la comunicación racional mutua" (Friedman, 2002: 185).

Esta forma de ver el asunto confunde lo que Devitt eficazmente había distinguido. La ruptura en la comunicación entre dos científicos que defienden 
teorías rivales es una contingencia histórica, resultado de las características del contexto donde se da la comunicación, así como de variables relativas a la personalidad de los participantes en el debate, su formación y su ideología entre otras. No es una consecuencia de la intraducibilidad de las teorías en cuestión, ya que dos científicos que defienden teorías mutuamente no intertraducibles pueden, en algunos casos, comunicarse con éxito.

Si las rupturas en la comunicación se entienden como consecuencia de los fallos de traducción, no es posible explicar los casos en que defensores de teorías intraducibles pueden comprenderse mutuamente. Se trata en todo caso de diferentes niveles de impacto de las estructuras semánticas en las conductas lingüísticas, pero no en términos que permitan reducir las dificultades de comunicación de los sujetos a los rasgos semánticos de los lenguajes que estos emplean. Una breve viñeta sobre la revolución copernicana puede ilustrar este punto. Puede afirmarse sin dificultad que en los debates entre Galileo y los defensores del sistema geocéntrico hubo toda clase de fallos de comunicación, malentendidos, diálogos inconducentes, argumentaciones circulares y estrategias retóricas espurias. Puede también concederse que en ciertos aspectos del debate la ausencia de acuerdos mínimos sobre los que justificar las decisiones de uno y otro bando condujo a callejones sin salida.

Si nos situamos a nivel de las prácticas comunicacionales, todas estas dificultades pueden asociarse a problemas de comunicación. Pero si nos situamos en el plano de la interpretación, los problemas de comunicación no fueron impedimento para que Galileo pudiera comprender el lenguaje geocéntrico y utilizar correctamente sus términos. Si por otro lado aceptamos que Galileo y sus adversarios estaban separados por lenguajes al menos parcialmente intraducibles, puede ofrecerse un balance análogo. Algunos de los debates en que Galileo se involucró muestran divergencias insalvables de comunicación, como el caso de Ludovico delle Colombe y su ataque a las consecuencias teológicas de la movilidad de la tierra ${ }^{10}$. Pero otros debates con los partidarios del sistema geocéntrico parecen tener un nivel de malentendido mucho menor, como es el caso del informe de los astrónomos del Observatorio Vaticano sobre los descubrimientos telescópicos de Galileo. Allí no parece

\footnotetext{
${ }^{10}$ Una revisión detallada de este episodio se halla en Shea y Artigas (2004).
} 
haber incomprensión entre las partes, sino más bien desacuerdos de naturaleza técnica sobre las implicaciones de las observaciones realizadas mediante el telescopio ${ }^{11}$. Si aceptamos que los modelos astronómicos geocéntrico y heliocéntrico representan lenguajes intraducibles, la cuestión es por qué Galileo podía comunicarse mejor con algunos defensores del sistema geocéntrico que con otros. Más allá de los detalles históricos del caso, resulta de interés mostrar cómo esto es posible si se consideran por separado los problemas de la traducción y los de la comunicación.

Dos lenguajes pueden presentar problemas de traducción, esto es, uno de ellos puede contener términos que no son traducibles al otro, sin que esto derive necesariamente en fallos de comunicación entre los hablantes que los utilizan. Esto en cuanto la traducción - exitosa o fallida- es una propiedad de las relaciones entre lenguajes, mientras que la comunicación -exitosa o fallida- es un proceso dado entre los sujetos que hablan un lenguaje. De aquí que dos sujetos puedan comunicarse, aunque hablen lenguajes intraducibles, del mismo modo que dos sujetos pueden tener problemas de comunicación aunque hablen lenguajes traducibles. Si nos situamos en el plano de las relaciones entre lenguajes, podemos incluso responsabilizar parcialmente a los fallos de traducción dados a nivel interlingüístico por los fallos de comunicación dados en el nivel intersubjetivo. Lo que resulta más difícil de afirmar es que los fallos de traducción necesariamente conducen a fallos de comunicación, o que todos los malentendidos se originan en fallos de traducción.

Una afirmación del estilo "Si A y B son teorías intraducibles, sus defensores tendrán problemas de comunicación” supone que las diferencias semánticas de las teorías no pueden neutralizarse en la interacción lingüística de los sujetos. Ello implica que las diferencias entre los lenguajes ponen un límite que no puede ser rebasado por las estrategias comunicacionales de los hablantes. Pero la revisión de casos parece ir en sentido opuesto: dadas dos teorías intraducibles, algunas interacciones lingüísticas entre sus defensores resultan fallidas y otras resultan exitosas. En los debates sobre el copernicanismo a principios del siglo XVII, Galileo se entendía con los aristotélicos en algunas ocasiones y en otras no. Al mismo tiempo Kepler casi siempre lograba en-

\footnotetext{
${ }^{11}$ Es de referencia para este episodio el trabajo de Fantoli (2003).
} 
tenderse con Tycho Brahe, a pesar de que defendían teorías incompatibles sobre el movimiento planetario. Durante la revolución química del siglo XVIII Priestley y Lavoisier mantenían varios desacuerdos, pero no parecen haber sufrido ningún problema de comprensión.

Las interacciones lingüísticas muestran una riqueza de estrategias y de recursos de comunicación que no puede reducirse a las relaciones de intraducibilidad de los lenguajes en juego. Partiendo de teorías intraducibles, en algunas ocasiones los sujetos fracasan al comunicarse y en otras logran interactuar con éxito. Supongamos que A y B son dos teorías intraducibles. $\mathrm{A}_{1}$ es un científico que defiende $\mathrm{A} ; \mathrm{y}_{1} \mathrm{y}_{2}$ son dos científicos que defienden $\mathrm{B}$. Puede darse el caso de que $\mathrm{A}_{1}$ no logre comunicarse con $\mathrm{B}_{1}$ y sí logre comunicarse con $\mathrm{B}_{2}$, siendo que ambos defienden la teoría $\mathrm{B}$. Esto puede deberse a varios factores: $B_{2}$ puede ser bilingüe, $B_{1}$ puede tener mayores resistencias que $\mathrm{B}_{2}$ a la teoría $\mathrm{A}$, o menos formación en sus conceptos, etc. El que la comunicación falle en un caso y tenga éxito en el otro puede deberse a una amplia serie de razones, pero no es una consecuencia automática del hecho de que $\mathrm{A}$ y B son intraducibles.

Hasta aquí he tratado de mostrar que en el contexto de la tesis kuhniana de la inconmensurabilidad, los problemas de la traducción, la interpretación y la comunicación refieren a procesos independientes, por lo que el mapeo de las divergencias entre dos teorías inconmensurables requiere considerar por separado estos tres niveles. Sin embargo, dicha consideración requiere ponderar la especificidad de cada plano sin establecer relaciones de inferencia entre uno y otro, cosa que no pocas veces ha sido frecuente en la discusión de las tesis de Kuhn.

\section{§6. CONCLUSIÓN}

Los problemas de la interpretación, la traducción y la comunicación aparecen frecuentemente ligados a las discusiones sobre la tesis de la inconmensurabilidad. Tanto en las primeras formulaciones de Kuhn como en varias de las críticas que recibió su propuesta, estos tres planos no aparecen claramente distinguidos, lo que llevó a considerar que las diferencias semánticas 
conducen necesariamente a problemas de traducción, o que los fallos de traducción comprometen la posibilidad de interpretación. La distinción kuhniana entre interpretación y traducción permitió echar algo de luz sobre este problema, mostrando que los fallos de traducción entre teorías son independientes de las estrategias interpretativas que utilizan los sujetos para aprender un lenguaje, por lo que la interpretación puede ser exitosa a pesar de que la traducción resulte fallida.

A partir de la distinción efectuada por Kuhn he defendido que es posible autonomizar aún más estos tres dominios lingüísticos; si se ubica los problemas de traducción en el nivel semántico, los problemas de interpretación en el nivel epistémico y los problemas de comunicación en el nivel de la práctica lingüística. Esto implica que la traducción es una relación semántica entre dos lenguajes, cuyo éxito depende de las características de los lenguajes en juego y no de las estrategias interpretativas empleadas por los hablantes de dichos lenguajes. La interpretación, por el contrario, sí depende de las estrategias que los sujetos despliegan para comprender un lenguaje, una vez que se trata de una relación epistémica entre un sujeto y un lenguaje. Finalmente, la comunicación puede ser considerada como una relación entre dos sujetos, que involucra las conductas lingüísticas que ambos desarrollan con el objetivo de comunicarse. Traducción, interpretación y comunicación son procesos independientes en cuanto se predican de unidades relacionales diferentes: la traducción se predica de la relación entre dos lenguajes, la interpretación refiere a la relación entre un sujeto y un lenguaje, y la comunicación a la relación entre dos sujetos.

Si se acepta que estas tres nociones se aplican a dominios diferentes no es posible establecer relaciones inferenciales entre ellas. Dos defensores de teorías rivales pueden presentar problemas de comunicación, pero estos no pueden ser inferidos de las diferencias semánticas entre las teorías en disputa. A efectos de clarificar los componentes y el alcance de los episodios de inconmensurabilidad, cada uno de estos procesos requiere una revisión por separado. En estos términos, la triple distinción entre interpretación, traducción y comunicación resulta fértil para delimitar de qué tipo de procesos puede predicarse la inconmensurabilidad, así como para no amplificar indebidamente la inconmensurabilidad kuhniana más allá del dominio lingüístico que le es propio. 


\section{REFERENCIAS BIBLIOGRÁFICAS}

Davidson, D. (1974). De la idea misma de un esquema conceptual. En De la verdad y de la interpretación (pp. 189-203). Barcelona: Gedisa, 1990.

Devitt, M. (1979). Against Incommensurability. Australasian Journal of Philosophy, 57(1), 29-50.

Falguera, J. L. (2012). Comparación epistémica de teorías inconmensurables. En P. Lorenzano y O. Nudler (eds.), El camino desde Kuhn. La inconmensurabilidad hoy (pp. 119-170). Madrid: Biblioteca Nueva.

Fantoli, A. (2003). Galileo: For Copernicanism and for the Church. Vatican City: Vatican Observatory/University of Notre Dame Press.

Favretti, R., Sandri, G., Scazzieri, R. (1999). Translating Languages: an Introductory Essay. In R. Favretti et al. (eds.), Incommensurability and Translation: Kuhnian Perspectives on Scientific Communication and Theory Change (pp. 1-29). Northampton, MA: Edward Elgar.

Friedman, M. (2002). Kant, Kuhn, and the Rationality of Science. Philosophy of Science, 69(2), 171-190.

Hesse, M. (1983). Comments on Kuhn's 'Commensurability, Comparability, Communicability. In PSA, Proceedings of the Biennial Meeting of the Philosophy of Science Association, v. 2: Symposia and Invited Papers (2), 704-711.

Hoyningen-Huene, P., Sankey, H. (2001). Introduction. In P. Hoyningen-Huene, H. Sankey (eds.), Incommensurability and Related Matters, VII-XXXIII. BSPS, 216, Dordrecht: Kluwer.

Kitcher, P. (1978). Theories, Theorists, and Theoretical Change. The Philosophical Review, LXXXVII (4), 519-547.

Kuhn, T. (1962). La Estructura de las Revoluciones Cientificas. México: FCE, 2013. Introducción de Ian Hacking. Traducción e introducción de Carlos Solís.

Kuhn, T. (1969). Epílogo: 1969. En La Estructura de las Revoluciones Cientificas. México: FCE, 2013. 
Kuhn, T. (1970). Consideraciones en torno a mis críticos. En El camino desde La estructura (pp. 151-209). Barcelona: Paidós, 2000.

Kuhn, T. (1974). Algo más sobre paradigmas. En La tensión esencial (pp. $317-$ 343). Madrid: FCE, 1993.

Kuhn, T. (1977). Objetividad, juicios de valor y elección de teoría. En La tensión esencial (pp. 344-364).

Kuhn, T. (1981). ¿Qué son las revoluciones científicas? En ¿Qué son las revoluciones cientificas? (pp. 55-93). Barcelona: Paidós, 1989.

Kuhn, T. (1983). Conmensurabilidad, comparabilidad, y comunicabilidad. En ¿Qué son las revoluciones científicas? (pp. 95-135). Barcelona: Paidós, 1989.

Kuhn, T. (1989). Mundos posibles en historia de la ciencia. En El camino desde la estructura (pp. 77-112). Barcelona: Paidós, 2000.

Laudan, L. (1996). Beyond Positivism and Relativism. Boulder: Westview Press.

Marcum, J. (2005). Thomas Kuhn's Revolution: An Historical Philosophy Of Science. New York: Continuum.

Mayoral, J. (2017). Mundos fenoménicos y léxicos científicos: El relativismo lingüístico de Thomas Kuhn. Revista de Filosofía, 42(1), 117-134.

Pérez Ransanz, A. R. (1999). Kuhn y el cambio cientifico. México: FCE.

Pérez Ransanz, A. R. (2012). Recuperando la inconmensurabilidad axiológica. En P. Lorenzano y O. Nudler (eds.), El camino desde Kuhn. La inconmensurabilidad hoy (pp. 367-382). Madrid: Biblioteca Nueva.

Putnam, H. (1974). The "Corroboration" of Theories. In R. Boyd, P. Gasper, J. Trout (eds.), The Philosophy of Science (pp. 121-137). Cambridge/ Mass.: MIT Press, 1991.

Putnam, H. (1981). Razón, verdad e historia. Madrid: Tecnos, 1988.

Putnam, H. (1982). Why reason can't be naturalized. En Realism and Reason, Philosophical Papers, vol. 3 (pp. 229-247). Cambridge/Mass.: Cambridge University Press, 1983. 
Rivadulla, A. (2012). La tesis de la inconmensurabilidad y el desarrollo de la física. En P. Lorenzano y O. Nudler (eds.), El camino desde Kuhn. La inconmensurabilidad hoy (pp. 383-398). Madrid: Biblioteca Nueva.

Sankey, H. (2000). The language of science: meaning variance and theory comparison. Language Sciences (22), 117-136.

Siegel, H. (1980). Objectivity, Rationality, Incommensurability, and more. British Journal for the Philosophy of Science, 31(4), 359-375.

Shea, W., Artigas, M. (2004). Galileo in Rome. New York: Oxford University Press. 